\title{
Iterative Solution of Relative Localization for Cooperative Multi-robot Using IEKF
}

\author{
Kyunghyun Lee, Hyungkwan Kwon, Kwanho You*
}

Department of Electrical and Computer Engineering, Sungkyunkwan University, South Korea

Copyright $\bigcirc 2017$ by authors, all rights reserved. Authors agree that this article remains permanently open access under the terms of the Creative Commons Attribution License 4.0 International License

\begin{abstract}
Recently, multi-robot systems are emerging in various industries. The multi-robot systems have several advantages in comparison with a single-robot system. The task of single-robot system is limited, because a bigger robot is required to perform more multiple functions. However, multi-robot systems can distribute the functions to each robot. The localization problem is essential for realization and it becomes more important in the multi-robot system. The multi-robot system needs to work maintaining a formation. In order to accomplish the given mission in the demanded formation, the localization is important. For multi-robot localization, we use the relative position to find robots' position based on odometry sensor, and the iterative Kalman filter algorithm is utilized to estimate the accurate position.
\end{abstract}

Keywords Multi-robot Localization, Formation, Relative Position, Odometry, Iterative Kalman Filter

\section{Introduction}

The mobile robots can be used in various fields, and the mobile robots' technology and devices are classified according to the applied areas. The localization problem is one of the most important techniques and a fundamental for a mobile robot system. We can track the path of mobile robot if the robot knows its exact position continuously. When the robot uses only the dead-reckoning technique based on an odometry sensor, the accuracy of localization is limited by accumulation of positioning error, slip of a robot's wheel, kidnap problem, etc. [1]. The kidnap problem means that a well-localized robot is teleported to other position without any notification. The kidnapped robot is relocated in a different position manually and it has to relocalize itself based on the new sensors.

The multi-robot cooperation system has attracted a lot of interest recently. The multi-robot system has some benefits. Each robot in the multi-robot system can be more downsized than a single robot system, and it has more various applications [2]. The security robot system is one of the examples. If the security robot system uses a single robot, the system works within the limited functions. However, multi-robot system can prevent many dangers such as gas leaks, theft, fire accident, etc. Also, the multi-robot system is efficient when they are installed against the invaders. The robots can be grouped to surround the intruders. In case of the multi-robot system, the localization problem becomes more important. If each robot does not know its own position and other robot's local information, the robots can collide and their duties become tough to complete. Also, the multi-robot localization has another problem, i.e., each robot should know others' location in real time [3].

The formation matters in the multi-robot system in order to accomplish their goal. In every situation, each single robot has its own mission which finally becomes the multi-robot system's ultimate goal. However, the odometry sensor has some critical issues caused by an error accumulation, wheel's slip, kidnap, etc. [4].

There have been numerous approaches to single robot localization. Most studies to solve the localization problem utilize the proprioceptive sensors such as encoders in the wheel and exteroceptive sensors such as beacons. However, the multi-robot localization researches are not relatively common. As a simple approach, N-robots localization using $\mathrm{N}$ times independent position estimation is considered as a multi-robot localization method. As the advantages of using multi-robot system recently attract many researchers, the localization for multi-robot system has been studied actively. The multi-robot localization using relative observations was proposed by Martinelli [5]. The relative observation is made with relative bearing, distance and orientation. Also, Roumeliotis [6] proposed a distributed multi-robot localization. The distributed localization method uses multiple Kalman filters and each Kalman filter operates on each separate robot. Burgard [7] proposed collaborative multi-robot exploration which uses grid map to probe in unknown environments. Karazume [8] also proposed the cooperative positioning system principle. In this principle, one robot stays while the others are moving, and the moving 
robots' locations are calculated on the basis of the stationary robot's position.

We propose the localization of multi-robot system using the relative position in this paper. Each robot's location which is gained from the odometry sensor is used to obtain the relative position. In order to compensate the errors of odometry sensor, we use IR sensor as measurement values, then we apply the iterative extended Kalman filter to this process to track the accurate paths of multi-robot.

This paper is organized as follows. In section 2, we introduce the system modeling for mobile multi-robot. The multi-robot localization algorithm which uses the relative position and the iterative extended Kalman filter are described in section 3, and the simulation results show the improved localization performance of the proposed algorithm in section 4. Conclusions are drawn in section 5.

\section{System Modeling of Multi-robot}

In this section, we introduce a kinematic model of a single robot using odometry sensor and relative position among multi-robot.

\subsection{Kinematic Model of a Mobile Robot}

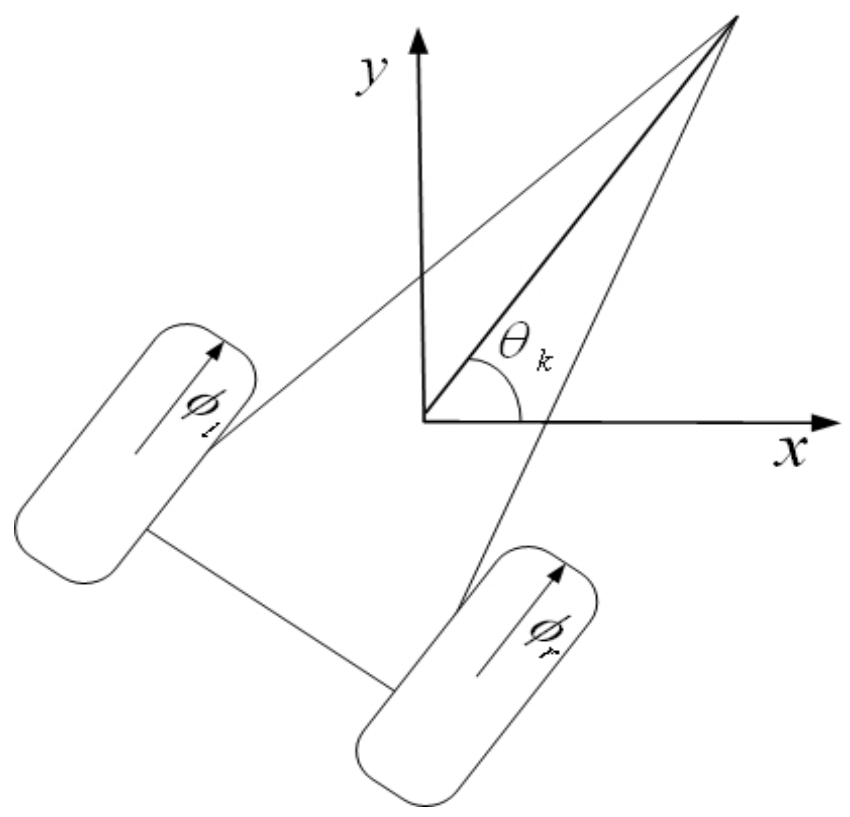

Figure 1. Kinematic model of a single robot

The wheeled mobile robot can be modeled on the basis of an odometry sensor. The model considers the robot's the x-y position and orientation in the global coordinate. The direction and position is calculated by movement of wheels. In figure 1 , the orientation is described as $\theta$ and $\phi_{r}$ and $\phi_{l}$ are the angular velocity of right and left wheel measured by the encoders, respectively. The well-known motion model of a robot is expressed as follows,

$$
\begin{aligned}
X_{k+1} & =X_{k}+T_{s} \frac{r}{2}\left[\phi_{r}+\phi_{l}\right] \cos \theta_{k}+\varepsilon_{X} \\
Y_{k+1} & =Y_{k}+T_{s} \frac{r}{2}\left[\phi_{r}+\phi_{l}\right] \sin \theta_{k}+\varepsilon_{Y} \\
\theta_{k+1} & =\theta_{k}+T_{s} \frac{r}{b}\left[\phi_{r}-\phi_{l}\right]+\varepsilon_{\theta} \\
p^{o d o} & =[X, Y, \theta]
\end{aligned}
$$

where $X, Y$ and $\theta$ are the $\mathrm{x}, \mathrm{y}$ position and the orientation of a robot using odometry in global coordinate, respectively. $T_{s}$ is the discrete sampling time, $r$ is the wheel radius, $b$ is the distance between wheel centers of the vehicle. $\varepsilon$ is a Gaussian white noise.

\subsection{Relative Position}

In the multi-robot system, the local positioning system is required in order to prevent collision and to find the right path. Also, the formation can be performed from the local positioning results. The relative position is one of the famous local positioning skills. Therefore, the relative position is most frequently used as a multi-robot localization technique. However, there are various ways to obtain the relative position.

The relative position configuration is shown in figure 2 and table 1. $r_{0}^{1}$ means the distance between robot $R_{0}$ and $R_{1}$, and $r_{0}^{2}, r_{0}^{3}, r_{0}^{4}$ are the distances between $R_{0}$ and each corresponding robot, respectively. Also, $r_{1}^{2}, r_{1}^{3}, r_{1}^{4}$ are the distances between $R_{1}$ and each corresponding $R_{2}$, $R_{3}$ and $R_{4}$, respectively. In this configuration, the relative position consists of the distance and angles, and the robot $R_{0}$ is considered as a center robot. Also, the angle is defined from a base line which is $\overline{R_{0} R_{1}}$.

We stipulate a relative position for a set of the mobile robot which this paper covers. First, we assume the following states:

1) A set of independent robots move in same altitude. The motion of each robot is described by its own odometry sensor. These sensors observe the self-motion of the robot;

2) All robots are equipped with communication unit in order to exchange its own position and orientation within the group;

3) The master robot carries exteroceptive sensor which measures respective distant and bearing of satellite robots. 


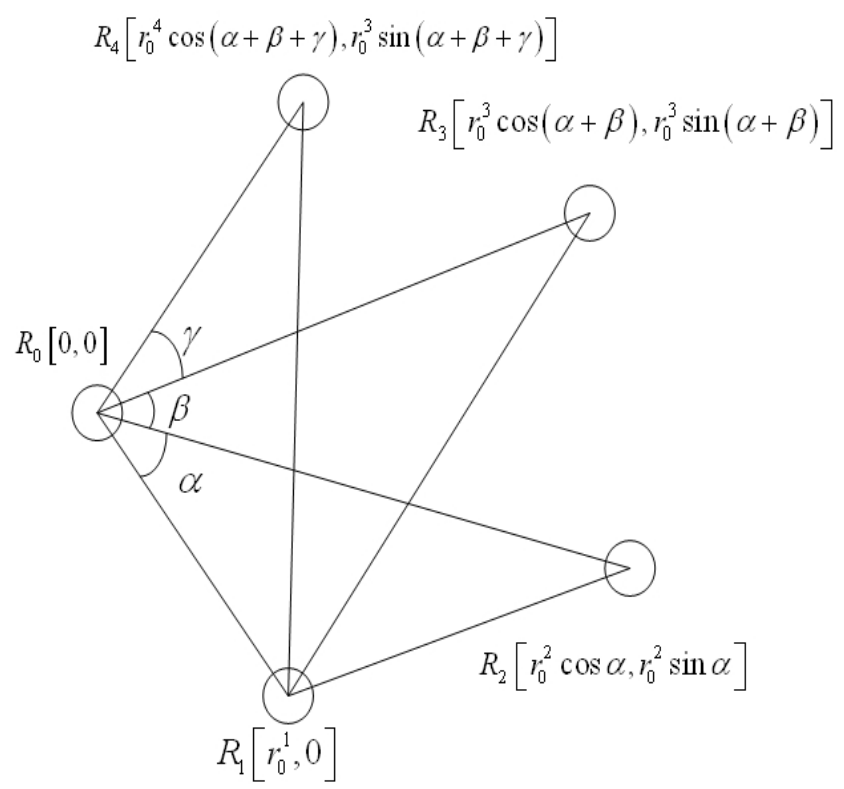

Figure 2. Cases of multi-robot localization

Table 1. Cases of multi-robot localization

\begin{tabular}{|c|c|}
\hline Case 1 & $R_{0}[0,0]$ \\
\hline Case 2 & $R_{1}\left[r_{0}^{1}, 0\right]$ \\
\hline Case 3 & $\alpha=\cos ^{-1}\left[\frac{\left(r_{0}^{1}\right)^{2}+\left(r_{0}^{2}\right)^{2}-\left(r_{1}^{2}\right)^{2}}{2 r_{0}^{1} r_{0}^{2}}\right]$ \\
\hline Case 4 & $R_{2}\left[r_{0}^{2} \cos \alpha, r_{0}^{2} \sin \alpha\right]$ \\
& $\alpha+\beta=\cos ^{-1}\left[\frac{\left(r_{0}^{1}\right)^{2}+\left(r_{0}^{3}\right)^{2}-\left(r_{1}^{3}\right)^{2}}{2 r_{0}^{1} r_{0}^{3}}\right]$ \\
Case 5 & $R_{3}\left[r_{0}^{3} \cos ^{2}(\alpha+\beta), r_{0}^{3} \sin (\alpha+\beta)\right]$ \\
& $R_{4}\left[r_{0}^{4} \cos \left(\alpha+\beta=\cos ^{-1}\left[\frac{\left(r_{0}^{1}\right)^{2}+\left(r_{0}^{4}\right)^{2}-\left(r_{1}^{4}\right)^{2}}{2 r_{0}^{1} r_{0}^{4}}\right]\right.\right.$ \\
\hline
\end{tabular}

When a robot firstly comes into the center robot's sensing range, the robot becomes $R_{1}$ and the relative position is obtained by $R_{1}\left[r_{0}^{1}, 0\right]$ which becomes a base axis line. Then the next robots presume as $R_{2}, R_{3}$ and $R_{4}$ in orders. If the $R_{3}$ comes within the range of $\alpha$, then the angle $\alpha$ is ignored in the case 4 and $\cos (\alpha+\beta)$ becomes $\cos \beta$. These angular values and distances are obtained by an odometry sensor. For this process, every robot has odometry sensors to estimate their own positions, and the kinematic model is used in the single robot positioning.

When a master robot and two satellite robots reach the sensible area, the relative position among the three robots can be described as following [9]

$$
r_{0}^{i}=h_{i}\left(x_{0}, x_{1}, x_{2}, y_{0}, y_{1}, y_{2}\right), i=1,2 .
$$

where $r_{0}^{i}$ represents the distance between the master robot and the satellite robot $R_{i} . x_{j}$ and $y_{j}$ are the position of $j$ -th robot in the global coordinate.

For data processing, the global coordinate needs to be transformed into a relative domain.

$$
\left[\begin{array}{l}
x_{r} \\
y_{r}
\end{array}\right]=\left[\begin{array}{cc}
\cos \varphi & \sin \varphi \\
-\sin \varphi & \cos \varphi
\end{array}\right]\left[\begin{array}{l}
x_{g} \\
y_{g}
\end{array}\right]+\left[\begin{array}{l}
\chi \\
\xi
\end{array}\right]
$$

where $\varphi$ is the angle between the relative axis and the global axis, $\left(x_{r}, y_{r}\right)$ is the position of the robot in the relative axis, $\left(x_{g}, y_{g}\right)$ is the position of the robot in the global axis, and $(\chi, \xi)$ is the difference between the relative coordinate and the global one.

\section{Iterative EKF Algorithm}

In this section, we discuss the iterative EKF which improves the performance of the multi-robot localization system. The iterative EKF has the iterative measurement update process which differs from the normal EKF. The iterative measurement update process provides fast convergence rate by the linearization of measurement value iteratively. Therefore, the iterative EKF has the same time update process as the normal one. The update process of the proposed algorithm is shown below,

1) Time Update (Prediction)

$$
\begin{aligned}
& \hat{x}_{k}^{-}=f\left(\hat{x}_{k-1}, u_{k}, \hat{w}_{k}^{-}\right) \\
& P_{x_{k}}^{-}=A_{k-1} P_{x_{k-1}} A_{k-1}^{T}+R^{V}
\end{aligned}
$$

2) Measurement Update (Correction)

$$
\begin{aligned}
\hat{x}_{k, 0}^{+} & =\hat{x}_{k}^{-} \\
P_{k, 0}^{+} & =P_{k}^{-} \\
C_{k, i} & =\left.\frac{\partial h}{\partial x}\right|_{\hat{x}_{k, i}^{+}} \\
K_{k, i} & =P_{k, i}^{-} C_{k, i}^{T}\left(C_{k, i} P_{k, i}^{-} C_{k, i}^{T}+R^{n}\right)^{-1} \\
P_{k, i+1}^{+} & =\left(I-K_{k, i} C_{k, i}\right) P_{k, i}^{-} \\
\hat{x}_{k, i+1}^{+} & =\hat{x}_{k}^{-}+K_{k, i}\left[y_{k}-h\left(\hat{x}_{k, i}^{+}\right)-C_{k, i}\left(\hat{x}_{k}^{-}-\hat{x}_{k, i}^{+}\right)\right] \\
\hat{x}_{k}^{+} & =\hat{x}_{k, N+1}^{+} \\
P_{k}^{+} & =P_{k, N+1}^{+}
\end{aligned}
$$

As shown in the equation (4), the time update has the same process as in the normal EKF. The $\hat{x}_{k}^{-}$means a priori 
estimation value and $P_{k}^{-}$represents the estimation error covariance. The equation (5) is the iterated process for the linearization. In the iteration process, $\hat{x}_{k, o}^{+}$and $P_{k, 0}^{+}$are used as $\hat{x}_{k}^{-}$and $P_{k}^{-}$. After $i$-th iteration, the posterior estimated state is represented as $\hat{x}_{k}^{+} . C_{k, i}$ means the Jacobian matrix at $x_{k}=\hat{x}_{k, i}^{+}$, and the Kalman gain of the proposed algorithm is $K_{k, i}$.

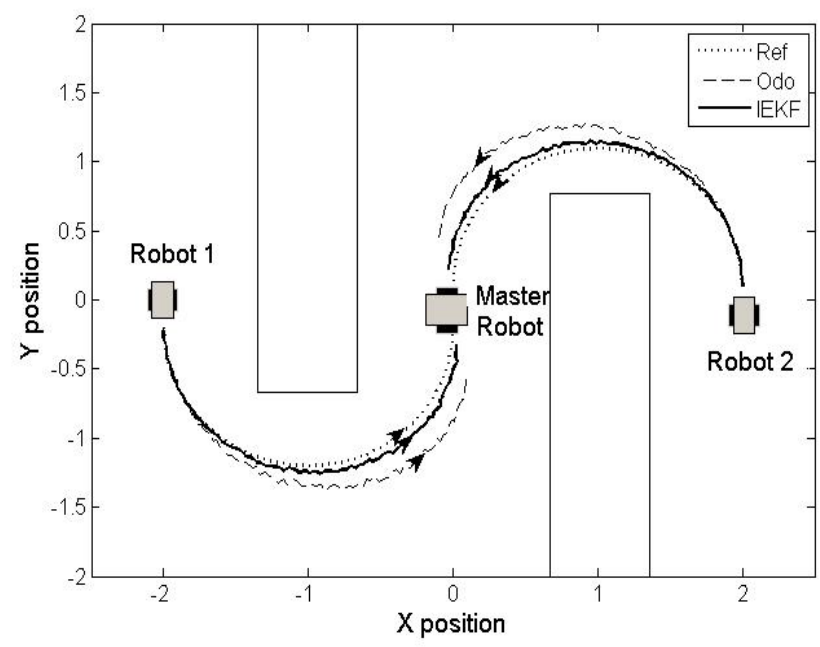

Figure 3. Simulation result of tracking performance

\section{Simulations}

To verify the effectiveness of proposed position estimation algorithm, a simulation of the multi-robot localization system is implemented. In this simulation, a master robot and two satellite robots compose the system. The master robot contains a communication host to exchange position information and an IR sensor to measure distance to others. The others are slaves which contain only IR transmitter as in [10].

Figure 3 shows a simulation result of tracking path. In figure 3, two satellite robots move forward the master robot on collision free path which is represented as the dotted line and set a formation. The dashed line means a tracking result with odometry sensor only and the solid line represents the compensation result. The satellite robots can move toward the precise position of the master robot without a collision through our proposed algorithm based on IEKF even if the master robot is kidnapped. Figure 4 is a comparison of errors between dead-reckoning method and the proposed algorithm. The dotted line is an error of dead-reckoning, and the solid line represents an error of the proposed algorithm. The coordinate $\mathrm{x}$ and $\mathrm{y}$ are distinguished by adding point marks. Through figures 3 and 4, the performance of the proposed algorithm is much better than dead-reckoning method.

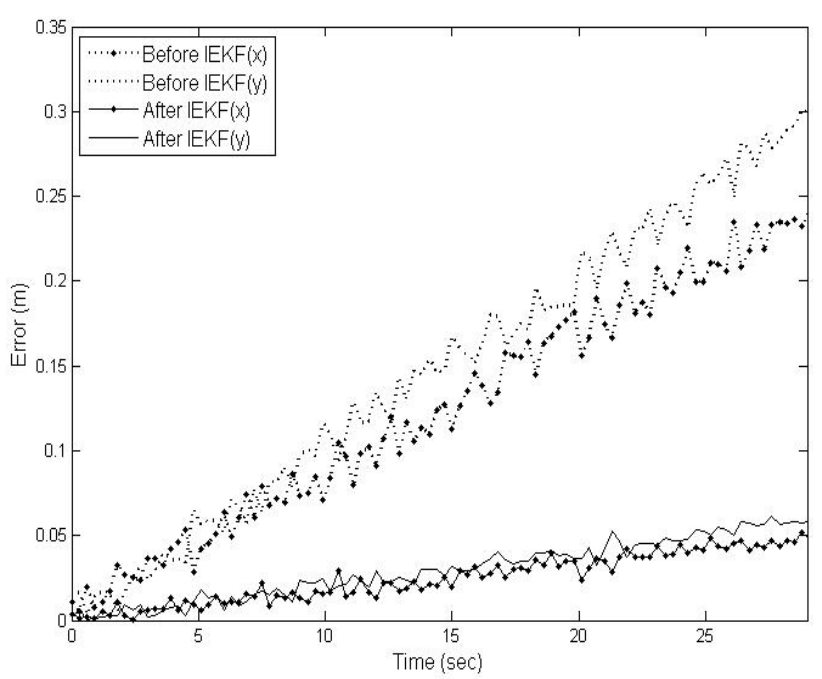

Figure 4. Error comparison

\section{Conclusions}

The localization problem becomes more significant issue in the multi-robot system. To reduce the influence of kidnap problem, we proposed a compensation method with a relative position model which is applicable for multi-robot system by applying the iterative extended-Kalman filter. With this algorithm, we improved the performance by reducing the tracking error. Also, our proposed algorithm overcame the issue of recovery from kidnap problem by using the computation of the current position adaptively. Through some simulations, it is demonstrated that the proposed algorithm can reduce the influence of kidnap problem and improve the tracking accuracy of multi-robot system.

\section{Acknowledgements}

This work was supported by the National Research Foundation of Korea(NRF) grant funded by the Korea government(MSIP) (NRF-2016R1A2B4011369).

\section{REFERENCES}

[1] H. Hardt, D. Wolf, and R. Husson. The dead reckoning localization system of the wheeled mobile robot ROMANE. IEEE/SICE/RSJ Int. Conf, Multi-sensor Fusion and Integration for Intelligent Systems 1996; 603-610.

[2] R. Alami, F. Robert, F. Ingrand, and S. Suzuki. Multi-robot cooperation through incremental plan-merging. IEEE Int. Conf. Robotics and Automation 1995; 3(1):2573-2579.

[3] A. Howard. Multi-robot Simultaneous Localization and Mapping using Particle Filters. The Int. Journal of Robotics Research 2006; 25(12):1243-1256. 
[4] B. Gerkey and M. Mataric. A Formal Analysis and Taxonomy of Task Allocation in Multi-Robot Systems. The Int. Journal of Robotics Research 2004; 23(9):939-954.

[5] A. Martinelli, F. Pont, and R. Siegwart. Multi-Robot Localization Using Relative Observations. IEEE Int. Conf. Robotics and Automation 2006; 2797-2802.

[6] S. Roumeliotis and G.A. Bekey. Distributed multirobot localization. IEEE Trans. on Robots and Automation 2002; 18(1):781-795.

[7] W. Burgard, M. Moors, D. Fox, R. Simmons, and S. Thrun. Collaborative multi-robot exploration. IEEE Int. Conf.
Robotics and Automation 2000; 476-481.

[8] R. Karazume, S. Nagata, and S. Hirose. Cooperative positioning with multiple robots, IEEE Int. Conf. Robotics and Automation 1994; 2(1):1250-1257.

[9] V. Kukshya, H. Krishnan, and C. Kellum. Performance Evaluation of a System for Estimating Relative Positions. IEEE Intelligent Vehicles Symposium 2006; 394-399.

[10] J. Pugh and A. Martinoli. Relative localization and communication module for small-scale multi-robot systems. IEEE Int. Conf. on Robotics and Automation 2006; 188-193. 